\title{
Factors that influence ultrasound evaluation of breast tumor size
}

\author{
Jin $\mathrm{Xu}^{1^{*}}$, Ge Ma ${ }^{2,3 *}$, Mengdi Liang², Yue Wang², Hong Pan², Li Li4, Cuiying Li', Wenbin \\ Zhou $^{2}$, Shui Wang',3
}

\begin{abstract}
*The authors share the first authorship
${ }^{1}$ Department of Breast and Thyroid Surgery, Nanjing First Hospital, Nanjing Medical University, Nanjing, ${ }^{2}$ Department of Breast Surgery, The First Affiliated Hospital with Nanjing Medical University, Nanjing, ${ }^{3}$ Jiangsu Key Lab of Cancer Biomarkers, Prevention and Treatment, Jiangsu Collaborative Innovation Center For Cancer Personalized Medicine, School of Public Health, Nanjing Medical University, Nanjing, ${ }^{4}$ Department of General Surgery, Huashan Hospital, Fudan University, Shanghai, ${ }^{5}$ Department of Ultrasound in Medicine, the First Affiliated Hospital with Nanjing Medical University, Nanjing, China
\end{abstract}

\begin{abstract}
Aims: To determine the factors influencing ultrasound breast tumor size assessment accuracy. Material and methods: Five factors (tumor type, molecular subtype, histological size, histological grade, and breast density) were used to assess the measurement accuracy of breast ultrasound in tumor size. Size underestimation was defined as ultrasound index lesion diameter $<$ histological size by at least $5 \mathrm{~mm}$. Results: Breast ultrasound underestimated tumor size significantly, especially in cases with intraductal components $(\mathrm{p}=0.002)$. There was a tendency for higher size underestimation in breast cancer tumors with high-histological grade ( $\mathrm{p}=0.03)$, human epidermal growth factor receptor type 2 (HER2)-overexpressing breast cancer tumors $(\mathrm{p}=0.02)$ and hormone receptor $(\mathrm{HR})-/$ HER $2+$ breast cancer tumors $(\mathrm{p}=0.008)$. Furthermore, core biopsy revealed higher probability of size underestimation with intraductal components $(\mathrm{p}=0.002)$. Size underestimation was more frequent with larger histological size $(\mathrm{p}<0.001)$. Masses in non-dense breasts were significantly underestimated $(\mathrm{p}=0.036)$ compared to dense breasts. Conclusions: The size underestimation was influenced by pathological type, molecular subtype, and histological size. The pathological results of core biopsy were conducive for predicting tumor size pre-surgery in precise breast cancer diagnosis.
\end{abstract}

Keywords: breast cancer; ultrasound; tumor size; molecular subtype; core biopsy

\section{Introduction}

Breast cancer is the most commonly diagnosed cancer in women. In recent years, breast-conserving surgery and minimally invasive treatment are becoming more appealing to women with early stage breast cancer [1]. Ul-

Received 08.11.2018 Accepted 31.01.2019

Med Ultrason

2019, Vol. 21, No 2, 144-151

Corresponding author: Shui Wang, MD

Department of Breast Surgery, The First Affili ated Hospital with Nanjing Medical University, 300 Guangzhou Road, 210029 Nanjing, China Phone: 0086-25-83718836 ext. 6456

Fax: 0086-25-83718836

E-mail:ws0801@hotmail.com trasonography (US) is a traditional technique for imaging breast masses that is advantageous in terms of its safety and usability. US measurement of breast cancer tumor size is a routine pre-surgery examination. The accurate measure of breast cancer tumor size is a precondition for individualized treatment. Previous studies have reported that tumor type and histological size are the key components associated with underestimation of tumor size [2-6] and the gland density does not affect the US evaluation of tumor size [7]. It has reported that the maximal tumor diameter assessed by US is within $5 \mathrm{~mm}$ of the pathologic tumor size in $79.8 \%$ of cases [4].

The molecular subtypes of breast cancer and core biopsy are widely used in individualized therapy [8]. However, to our knowledge, the relationship between US measurement of tumor size and molecular subtyping or 
the pathological results of core biopsy has not been reported.

In the present study, we enrolled a large population of patients with breast cancer to determine the factors that influence the accuracy of US breast tumor size assessment, especially the molecular subtyping.

\section{Material and methods}

\section{Inclusion and exclusion criteria}

We enrolled in this retrospective analysis 1028 patients with primary breast cancer who had underwent surgery in Jiangsu Breast Disease Center, the First Affiliated Hospital with Nanjing Medical University between January 2011 to June 2015 . The study was approved by local Ethics Committee. The inclusion criteria were: 1) US and pathological information could be obtained and reviewed in the medical inquiry system; 2) no local treatment before surgery. The exclusion criteria were as follows: 1) neoadjuvant chemotherapy prior surgery; 2) multiple lesions that could not be distinguished in the US or pathological reports; 3) mass with inhomogeneous echo or microcalcification spots without low echo area. In figure 1 the flow diagram of analysis process is detailed.

\section{Tumor size measurement in $U S$}

US was performed using a 3-14 MHz linear transducer (iU22; Philips Medical Systems, Bothell, WA, USA and MyLab Twice (Esaote S.p.A., Genova, Italy). Patients were required to lie in a supine position with their breasts exposed when the ultrasound was performed. All examinations and evaluations were made by a radiologist with more than 10 years of experience. A single low echo area was identified as the index lesion. Every controversial focus was diagnosed by two radiologists. The index lesion was sorted using the Breast Imaging Reporting and Data System (BI-RADS) and measured in three dimensions with US (fig 2). With the patient in the proper position, the radiologist scanned the breast in general to determine the location of the mass and the largest diameter of the tumor was measured, which was considered as the longest axis. The methods of the tumor size measurements are referred to in the previous study of our research group $[1,9]$. The halo or the edges of the speckles were included in the measurements of ultrasound $[10,11]$. If the observation error of three measurements was larger than $5 \mathrm{~mm}$, the measurement was conducted and determined by another senior radiologist until the error was less than $5 \mathrm{~mm}$. Then the three measures were averaged and a note as to the sonographic limits per lesion.

\section{Classification of breast density}

Mammography was performed using two digital fullfield instruments (Senographe 2000D, GE, Fairfield,

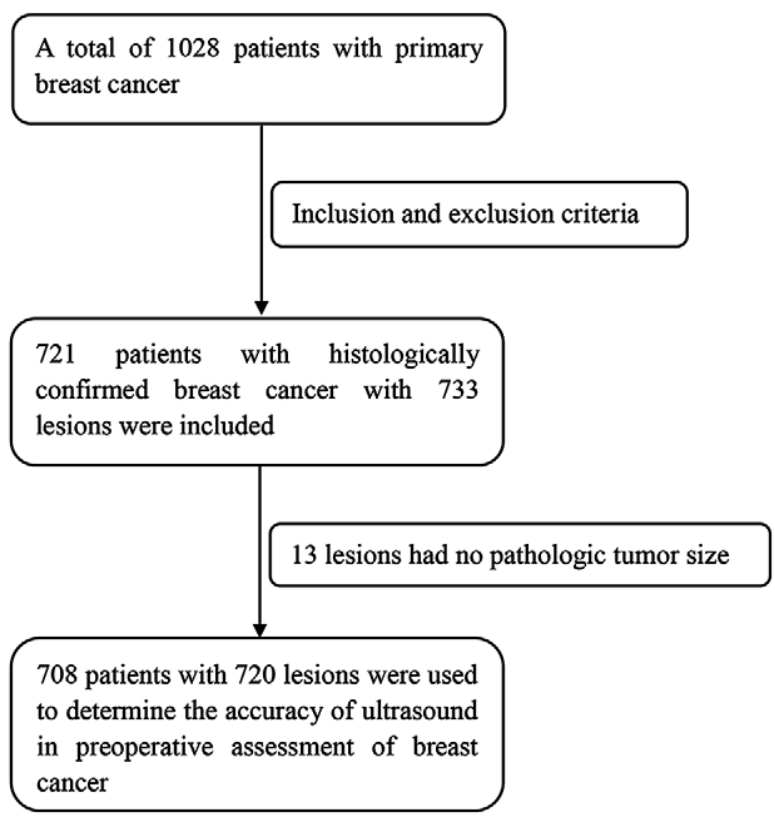

Fig 1. Flow diagram of screened and excluded patients

USA). The breast density was categorized according to the 4th edition of the BI-RADS proposed by the American College of Radiology (ACR). We divided patients into two groups according to different breast density, to evaluate if breast density was an influencing factor of US evaluation of breast tumor size.

\section{Core biopsy}

Core biopsy was performed with an automated Bard Magnum gun and a 14-gauge needle under ultrasound guidance. Based on the core biopsy pathological reports, the cases were divided into four groups: atypical ductal hyperplasia, pure ductal carcinoma in situ (DCIS), invasive carcinoma (IC, including pure IC [pIC] and a mixture of IC and DCIS) and other types.

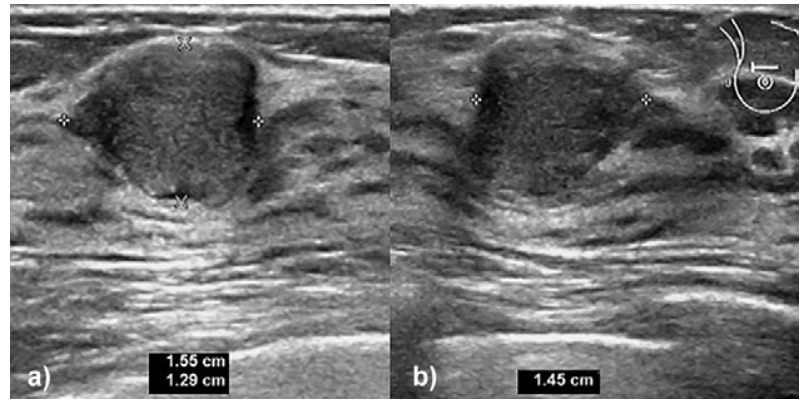

Fig 2. Ultrasonography (linear transducer with a frequency of 3-14 MHz) of a mass in 10 o'clock position in the right breast shows a hypoechoic irregular mass. The breast tumor size was measured in three dimensions: a) two major axes perpendicular to each other: b) the third major axis perpendicular to the previous two. 


\section{Tumor size measurement in pathology}

After surgery, the breast specimen was sliced sequentially into $5 \mathrm{~mm}$ sections perpendicular to the long axis of the tumor involving the treated tumor and adjacent tissue. The pathological measurements were in terms of the microscopic borders of the tumors. The histological size was $5 \mathrm{~mm} \times$ number of slices containing tumor cells. The maximum dimension of the mass in US was compared with the largest diameter in histology.

\section{Classification of postoperative pathological types}

Based on the postoperative pathological reports, the tumor types were divided into four groups: ductal carcinoma in situ (DCIS), invasive ductal carcinoma (IDC), invasive lobular carcinoma (ILC) and other tumors (including mucinous carcinoma and papillary carcinoma). The IDC cases were divided into two groups: IDC with intraductal components (IDC+DCIS) and pure IDC. Hormone receptor (estrogen receptor [ER] and progesterone receptor $[\mathrm{PR}]$ ) status, as well as human epidermal growth factor receptor type 2 (HER2) status, has guided breast cancer subtype grouping. In this study, 638 cases with complete ER and PR status and HER2 expression level were divided into three classic groups: HR+HER2-/+ (ER+, PR-/+, HER2-/+), HR-HER2- (ER-, PR-, HER2-), and HR-HER2+ (ER-, PR-, HER2+). The Scarff-Bloom-Richardson system was applied to classify the histological grade $[12,13]$.

Definition of overestimation and underestimation

Size underestimation was defined as image index lesion diameter $<$ histological size by at least $5 \mathrm{~mm}$. Overestimation was defined as image index lesion diameter $>$ histological size by at least $5 \mathrm{~mm}$. The measurement was defined as precise when the deviation between image and histology was $<5 \mathrm{~mm}$.

\section{Statistical analysis}

Results were expressed as mean \pm standard deviation (SD). For all cases, the largest diameter of a tumor was used as the size reference in both the imaging and pathological reports. The mean difference between ultrasound and histology of the various groups was calculated, and

Table I. Correlation between the accuracy of ultrasound evaluation and pathological size. A difference between ultrasound and pathological size $<5 \mathrm{~mm}$ was defined as accurate. A difference $>+5 \mathrm{~mm}$ was considered overestimation and $<-5 \mathrm{~mm}$ was considered underestimated.

\begin{tabular}{|c|c|c|c|c|c|}
\hline \multirow{2}{*}{ Factor } & \multirow{2}{*}{ Total } & \multicolumn{3}{|c|}{ Accuracy of ultrasound measurement } & \multirow{2}{*}{ p-value } \\
\hline & & Underestimated & Accurate & Overestimated & \\
\hline Total & 720 & $191(27 \%)$ & $406(56 \%)$ & $123(17 \%)$ & \\
\hline Tumor type & 720 & & & & 0.002 \\
\hline DCIS & 70 & 26 & 30 & 14 & \\
\hline Pure IDC & 421 & 103 & 253 & 65 & \\
\hline IDC+DCIS & 156 & 47 & 82 & 27 & \\
\hline ILC & 14 & 10 & 4 & 0 & \\
\hline Other & 59 & 31 & 10 & 18 & \\
\hline Histological grade & 564 & & & & 0.013 \\
\hline I and II & 304 & 64 & 193 & 47 & \\
\hline III & 260 & 83 & 140 & 38 & \\
\hline ER status & 712 & & & & 0.325 \\
\hline Positive & 553 & 139 & 320 & 94 & \\
\hline Negative & 152 & 47 & 79 & 26 & \\
\hline PR status & 704 & & & & 0.454 \\
\hline Positive & 469 & 117 & 270 & 82 & \\
\hline Negative & 235 & 69 & 128 & 38 & \\
\hline HER2 status & 638 & & & & 0.02 \\
\hline Positive & 160 & 58 & 74 & 28 & \\
\hline Negative & 478 & 111 & 291 & 76 & \\
\hline Ki-67 & 692 & & & & 0.67630 \\
\hline Low & 189 & 52 & 101 & 36 & \\
\hline High & 503 & 133 & 286 & 84 & \\
\hline Molecular subtype & 638 & & & & 0.008 \\
\hline HR+HER2-/+ & 492 & 125 & 288 & 79 & \\
\hline HR-HER2- & 89 & 20 & 57 & 12 & \\
\hline HR-HER2+ & 57 & 24 & 20 & 13 & \\
\hline
\end{tabular}

HER2 - human epidermal growth factor receptor type 2; HR - hormone receptor; IC - invasive carcinoma; DCIS - ductal carcinoma in situ; IDC - invasive ductal carcinoma; ILC - invasive lobular carcinoma; ER - estrogen receptor; PR - progesterone receptor 
the t-test was performed. Univariate analysis was carried out using the chi-square test or Fisher's exact test. In all cases, $\mathrm{p}$-values were two-tailed, and $\mathrm{p}$-values $<0.05$ were considered statistically significant. All statistical analyses were performed using SPSS version 21.0 (SPSS, IBM; Chicago, IL, USA).

\section{Results}

\section{Overall performance}

The 708 patients screened out from 1028 patients with 720 lesions were included to evaluate the accuracy of ultrasound in preoperative tumor size assessment of breast cancer.

The mean age of the included 708 patients was 51.80 years (median 50 years, range 25-93 years). The mean pathological tumor size was $23.4 \pm 13.2 \mathrm{~mm}$. The mean difference between the US and histological size was -2 $\mathrm{mm}(\mathrm{p}=0.002)$. In Table I the number of different tumor type and accuracy of ultrasound measurement is detailed.

The mean breast cancer tumor size discrepancy of the pure IDC cases between US and histology was -1.6 $\mathrm{mm}(22.8 \mathrm{~mm}$ vs. $21.2 \mathrm{~mm}, \mathrm{p}<0.01)$. The mean tumor size discrepancy was $-4.6 \mathrm{~mm}(25.5 \mathrm{~mm}$ vs. $20.9 \mathrm{~mm}$, $\mathrm{p}<0.01)$ and $-2.4 \mathrm{~mm}(25.5 \mathrm{~mm}$ vs. $23.1 \mathrm{~mm}, \mathrm{p}<0.01)$ for the DCIS and IDC+DCIS cases, respectively. At -5 $\mathrm{mm}$ level, DCIS cases showed the highest ultrasound underestimation.

A total 564 IDC tumors (including pure IDC and IDC+DCIS) were divided into two groups according to histological grade. The mean size discrepancies in low and high histological grade compared with US were $-0.99 \mathrm{~mm}$ and $-3.02 \mathrm{~mm}$, respectively $(\mathrm{p}=0.003)$. At -5 $\mathrm{mm}$ level, there were more underestimated cases in the high histological grade group than in the low histological grade group $(\mathrm{p}=0.010)$.

Table I lists the factors influencing misestimation. At $\pm 5 \mathrm{~mm}$ level, tumor type $(\mathrm{p}=0.002)$, histological grade $(p=0.010)$, HER2 status $(p=0.02)$ and molecular subtype $(p=0.008)$ significantly influenced US measurement. There was no evidence that ER status $(p=0.325)$, PR status $(p=0.454)$, or Ki-67 value $(p=0.676)$ could be used to predict US measurement alone.

As the largest group of breast cancer, the group of pure IDC was analyzed separately and this subgroup analysis suggested the same result. At $\pm 5 \mathrm{~mm}$ level, his-

Table II. Correlation between the accuracy of ultrasound evaluation and pathological size in the group of pure IDC. A difference between ultrasound and pathological size $<5 \mathrm{~mm}$ was defined as accurate. A difference $>+5 \mathrm{~mm}$ was considered overestimation and $<-5 \mathrm{~mm}$ was considered underestimated.

\begin{tabular}{|c|c|c|c|c|c|}
\hline \multirow{2}{*}{ Factor } & \multirow{2}{*}{ Total } & \multicolumn{3}{|c|}{$\begin{array}{c}\text { Accuracy of ultrasound measurement } \\
\end{array}$} & \multirow{2}{*}{ p-value } \\
\hline & & Underestimated & Accurate & Overestimated & \\
\hline Total & 421 & $103(24.5 \%)$ & $253(60.1 \%)$ & $65(15.4 \%)$ & \\
\hline Histological grade & 394 & 101 & 238 & 55 & 0.048 \\
\hline I and II & 208 & 43 & 136 & 29 & \\
\hline III & 186 & 58 & 102 & 26 & \\
\hline ER status & 415 & 100 & 252 & 63 & 0.871 \\
\hline Positive & 314 & 74 & 193 & 47 & \\
\hline Negative & 101 & 26 & 59 & 16 & \\
\hline PR status & 415 & 100 & 252 & 63 & 0.915 \\
\hline Positive & 265 & 62 & 162 & 41 & \\
\hline Negative & 150 & 38 & 90 & 22 & \\
\hline HER2 status & 392 & 94 & 243 & 55 & 0.025 \\
\hline Positive & 79 & 27 & 39 & 13 & \\
\hline Negative & 313 & 67 & 204 & 42 & \\
\hline Ki-67 & 412 & 101 & 246 & 65 & 0.712 \\
\hline Low & 96 & 25 & 54 & 17 & \\
\hline High & 316 & 76 & 192 & 48 & \\
\hline Molecular subtype & 392 & 94 & 243 & 55 & 0.021 \\
\hline HR+HER2-/+ & 293 & 69 & 184 & 40 & \\
\hline HR-HER2- & 70 & 13 & 49 & 8 & \\
\hline HR-HER2+ & 29 & 12 & 10 & 7 & \\
\hline Histological tumor size (mm) & 421 & 103 & 253 & 65 & \\
\hline$<20$ & 229 & 18 & 172 & 39 & \\
\hline $20-50$ & 181 & 74 & 81 & 26 & \\
\hline$>50$ & 11 & 11 & 0 & 0 & \\
\hline
\end{tabular}

HER2 - human epidermal growth factor receptor type 2; HR - hormone receptor; ER - estrogen receptor; PR - progesterone receptor 
tological grade $(\mathrm{p}=0.048)$, HER2 status $(\mathrm{p}=0.025)$, and molecular subtype $(\mathrm{p}=0.021)$ significantly influenced ultrasound measurement (Table II).

US-guided core biopsy was performed in 489 of 720 lesions. Of the 489 lesions, 55 were atypical ductal hyperplasia. The remaining 434 lesions were divided into three groups: DCIS, IC, and other types. Of these, 52 lesions $(11.98 \%)$ were DCIS; 376 cases $(86.64 \%)$ were IC, of which 315 cases (72.58\%) were pIC and 61 cases (14.06\%) were IC with intraductal components (IC+DCIS). Six cases (1.38\%) were other type (such as mucinous carcinoma). According to the $\pm 5 \mathrm{~mm}$ level, biopsy pathology reports indicated that breast cancer with a higher DCIS component was more likely to be underestimated, which was consistent with the results of the follow-up surgical pathological reports (fig 3). Given the limitation of tissue sampling in core biopsy, nearly half $(25 / 52,48.1 \%)$ of the DCIS cases were upgraded to invasive cancer after surgery. According to $\pm 5 \mathrm{~mm}$ level, the underestimated cases were 11/27 in DICS, 14/25 in DCIS upgrade. The results show no difference in the accuracy of US between the DCIS group and the DCIS-upgrade group $(\mathrm{p}=0.628)$.

\section{Impact of histological tumor size}

The results comparing image size to pathological size of the different tumor staging are presented in fig 4. A total of 387 cases had tumor size $<20 \mathrm{~mm}$ on US. The mean tumor size derived from ultrasound was $16.60 \mathrm{~mm}$, while the mean histological size was $14.61 \mathrm{~mm}(\mathrm{p}<0.01)$. In comparison to the overestimation of small tumors, US obviously underestimated tumor size in the patient group with higher stage tumors. The mean difference between US measured tumor size and pathological measured tumor size was $-4.55 \mathrm{~mm}(\mathrm{p}<0.01)$ and $-33.61 \mathrm{~mm}$ $(\mathrm{p}<0.01)$ in group II (histological size between 20-50 $\mathrm{mm}$ ) and group III (histological size $>50 \mathrm{~mm}$ ) tumors, respectively. Table III shows the consistency of US estimation when tumor pathological stage increased. US measurement tended to overestimate the size of small tumors while tending to underestimate the size of large tumors. According to the $\pm 5 \mathrm{~mm}$ level, ultrasound underestimated 33/387 cases (8.53\%) in group I (histological size $<20 \mathrm{~mm}), 135 / 309$ cases $(43.69 \%)$ in group II, and $23 / 24$ cases $(95.83 \%)$ in group III $(p<0.001)$. In the subgroup analysis of pure IDC, ultrasound underestimated $18 / 229$ cases $(7.86 \%)$ in group I (histological size $<20$ $\mathrm{mm}), 74 / 181$ cases $(40.88 \%)$ in group II, and $11 / 11$ cases $(100.00 \%)$ in group III $(\mathrm{p}<0.001)$.

\section{BI-RADS classification of breast tumors}

Based on the BI-RADS classification, 518 tumors were divided into five groups. According to the $\pm 5 \mathrm{~mm}$ level, 3/8 (37.5\%) BI-RADS 3, 19/48 (39.58\%) BI-RADS

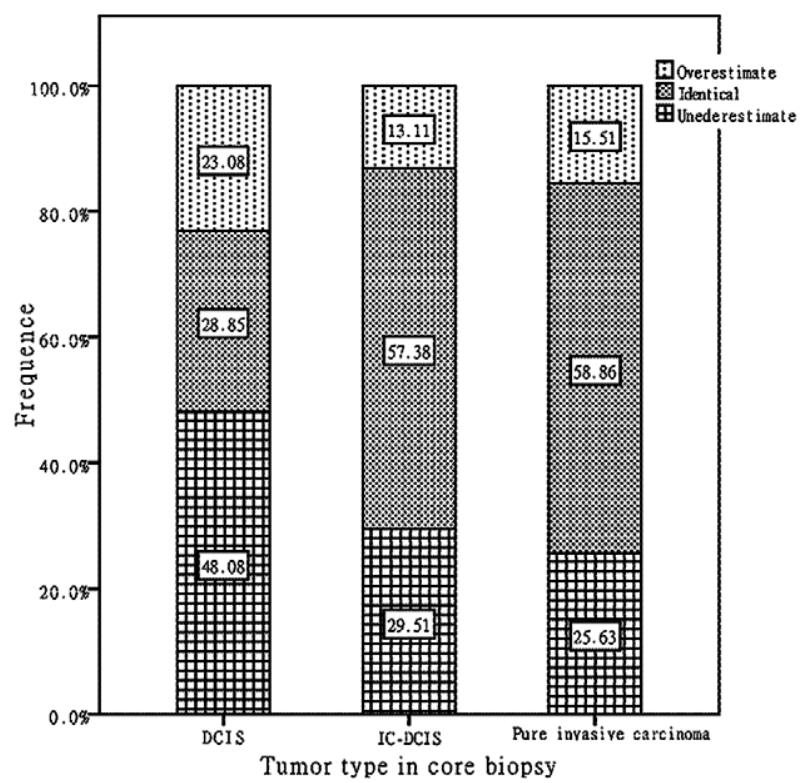

Fig 3. Percentage of underestimation in tumor types according to core biopsy results. According to the $\pm 5 \mathrm{~mm}$ level, the higher the DCIS component, the more likely it is to be underestimated $(\mathrm{p}=0.002)$.

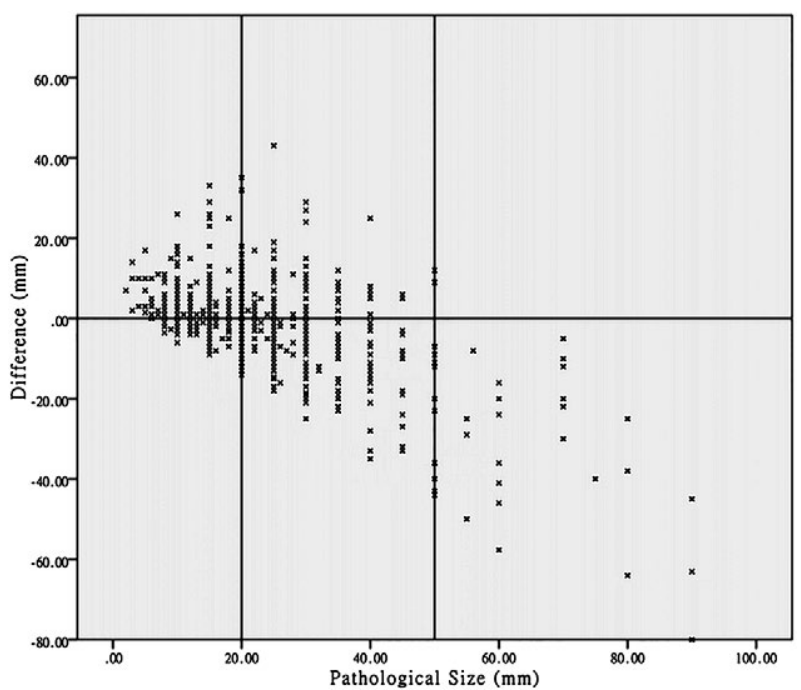

Fig 4. Scatter plot of the relationship between pathological size and difference between ultrasound and histology sizes for all tumors.

4A, 18/61 (29.51\%) BI-RADS 4B, 38/193 (19.69\%) BIRADS 4C, and 50/208 (24.04\%) BI-RADS 5 were underestimated. According to the $\pm 5 \mathrm{~mm}$ level, there was no significant difference between the BI-RADS 4B, 4C, and 5 groups $(\mathrm{p}=0.387)$. (Table IV).

\section{Breast density}

In this study, mammography was performed on 373 patients in our hospital. Masses in non-dense type (ACR 
Table III. The accuracy of ultrasound evaluation in different groups of the histological size

\begin{tabular}{llcl}
\hline Ultrasound size (mm) & \multicolumn{3}{c}{ Histologic size $(\mathbf{m m})$} \\
\hline & $\leq 20$ & $20-50$ & $\geq 50$ \\
\cline { 2 - 4 }$\leq 20$ & 301 & 101 & 6 \\
$20-50$ & 84 & 198 & 14 \\
$\geq 50$ & 2 & 10 & 4 \\
\hline
\end{tabular}

$1-3$ ) breasts were significantly underestimated $(\mathrm{p}=0.036)$ compared to dense type (ACR 4) breasts (fig 5). Age and menopausal status, which are the factors that may affect female breast density, had no effect on the evaluation of ultrasonography accuracy.

\section{Discussions}

Methodologically, US is subjective to some extent. In addition, it is difficult to ensure that the measurement of pathological specimens is determined based on three-dimensional US measurements. To address these issues, in this study, every dimension of the exponential lesion was measured three times by the same radiologist to minimize the error. In the comparison of pathology and US results, the two largest diameters were chosen to partially reduce the non-correspondence of pathology and US results.

Regarding non-mass lesions, Yang et al [14] reported that US did not help to characterize the morphology or extent of calcification in symptomatic DCIS. In our study, non-tumor lesions showed microcalcifications and more frequently destroyed echoes of the gland under US imaging, which was related to the pathological type of DCIS.

In this study, the difference between US imaging and histology reports was $-2 \mathrm{~mm}$. The corresponding data in other studies were $-8 \mathrm{~mm}$ and $-4.2 \mathrm{~mm}$, respectively $[2,15]$. A reduction in the underestimation of tumor size may be related to equipment upgrades. Another possible explanation is that, as a strong subjective test, US has been popularized in developing countries such as China in the past decades, enriching the experience of ultrasound doctors.

Regarding various tumor types, other studies have calculated linear regression between ultrasound measurements and pathological size of different tumor types, and

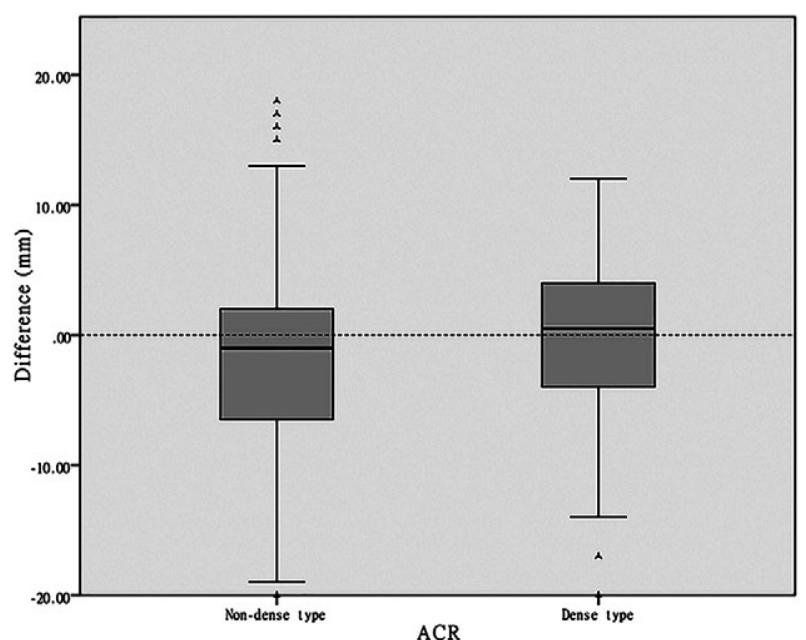

Fig 5. Masses in non-dense type (ACR 1-3) breasts were significantly underestimated compared to dense type (ACR 4) breasts.

ranked the degree of underestimation as IDC $<$ IDC+ILC $<$ ILC [15]. We found that underestimation of size was common for DCIS components cases. Satake et al [16] attributed the size underestimation to deformation of the breast tissue larger than the caliber and the fibrosis around the catheter involved may cause structural distortion on the ultrasound. However, there was no significant difference in pathology and imaging for the ILC group. The likely cause may be that the sample size of this type of tumor in our study is small.

The assertion that histological grade is an independent prognostic factor dates back to 1991 [13]. However, few researchers have determined the relationship between histological grade and US imaging. In this study, high-grade malignancies were associated with underestimation of the severity of low-grade malignancies. Highly malignant tumors can cause irregular shapes and fuzzy echoes, which can adversely affect the measurement of tumor size.

Perou et al [17] referred to the concept of molecular subtype of breast cancer in 2000, which proved to be a milestone in the progress of breast cancer treatment. Studies have increasingly shown that molecular biological markers such as ER, PR, and HER2 are closely related to the biological characteristics of cancer. The main

Table IV. Correlation between the accuracy of ultrasound evaluation and BI-RADS category. A difference between ultrasound and pathological size $<5 \mathrm{~mm}$ was defined as accurate. A difference $>+5 \mathrm{~mm}$ was considered overestimation and $<-5 \mathrm{~mm}$ was considered underestimated.

\begin{tabular}{llll}
\hline \multirow{2}{*}{ Ultrasound classification $(\mathbf{n = 5 1 8})$} & \multicolumn{3}{c}{ Accuracy of ultrasound measurement } \\
\cline { 2 - 4 } & Underestimated & Identical & Overestimated \\
\hline BI-RADS 3/4A $(\mathrm{n}=56)$ & 22 & 29 & 5 \\
BI-RADS 4B/4C/5 $(\mathrm{n}=462)$ & 106 & 263 & 93 \\
\hline
\end{tabular}


goal of this study was to explore the accuracy of tumor size measurements in different molecular biology states. The results showed that the size of HER2+ cases was underestimated, but no similar phenomenon was found in $\mathrm{ER}+$ or PR+ cases. Wojcinski et al [18] reported that HER2+ showed a high rate of structural deformation in surrounding tissues; this interpretation is consistent with our results. In clinical experience, breast cancer cases usually include three subtypes: cavity, HER2 overexpression and triple negative, although more detailed classification methods are gradually accepted. Our data indicate that the HER2 overexpression subtype clearly exhibits US underestimation compared to the other two subtypes. The underestimation of size can be attributed to the fuzzy boundary caused by the greater degree of HER 2 overexpressing tumor infiltration. It was noted that HER2 positive was statistically significantly associated with tumor vasculature [19]. However, in some indicators related to prognosis or tumor cell biological behavior, the HER2 overexpression subtype is expected to fall between the other two subtypes, while the triple negative subtype is associated with a poor prognosis [20,21]. In addition, triple-negative breast cancer is not a single entity but a series of different diseases [22]. The heterogeneity of triple-negative breast cancer can explain the results to some extent. Due to the complexity of triple-negative breast cancer, a prospective study of the agreement between US features and molecular subtypes is expected.

As a common means of preoperative diagnosis, the 2015 European Society of Cancer Surveillance Breast Cancer Clinical Practice Guide [23] requires a core biopsy. There are differences in pathology reports between core biopsy and subsequent surgery. In addition, the pathology report of the core biopsy is important for selecting the appropriate surgical procedure. Based on the results of the core biopsy, we divided patients into three groups and obtained similar results. The DCIS components are also a key factor in underestimating the size. It is worth mentioning that core biopsy is susceptible to inherent sampling errors, resulting in underestimation [24]. We also studied the factors of pathological escalation and did not find evidence that the DCIS histological escalation of the core biopsy would lead to further underestimation of ultrasound imaging. Taking into account the above two points, we have reason to believe that the use of core biopsy pathology report will facilitate accurate measurement of tumor size before surgery. At the same time, due to the characteristics of DCIS, if the results of the core biopsy include DCIS components, we should use some other image examinations, such as magnetic resonance imaging (MRI), before breast-conserving surgery.
In addition to the pathological type, histological size is also a key influencing factor. For small lesions $(<2$ $\mathrm{cm}$ ), we detected an overestimation of approximately 2 $\mathrm{mm}(\mathrm{p}<0.01)$. Pathological methods can explain almost indistinguishable deviations. Usually, the difference is small and may not have significant clinical value. However, we can conclude that tumor size is accurate or not underestimated in ultrasound examination. At the same time, as the size of histology increases, more sizes are underestimated. Others authors reported similar results $[3,5]$. Severe underestimation in large size cases may be associated with limitations of US probes and broader infiltration of advanced tumors.

BI-RADS classification is a recognized principle in the evaluation of breast lesions. Category 3 and $4 \mathrm{~A}$ are often benign lesions. Interestingly, low-grade tumors (supposed to be breast cancer) were underestimated. A possible explanation is that some of the malignant features, including malignant halos, are ignored in these misdiagnosed cases.

Breast density has been reported to be associated with breast mass sensitivity in a mammography but not with US or MRI [7]. According to the fourth edition of BI-RADS proposed by ACR, this study classifies female breasts into four categories. In dense breast tissue (ACR 4 ), the extent of the disease is slightly too high and the size of the non-compact breast tissue (ACR 1-3) is underestimated. The reason for this difference is that various breast tissue densities exhibit different background imaging and affect the measurement of breast lesions. No significant differences were found when age and menopausal status were used as the basis for classification.

\section{Limitations}

Although the diameters were measured in three radial directions in both the US and pathological specimens, only the largest diameter was compared in this study. Therefore, in a few cases, the diameters of the US and pathological specimens become unequal. We tried to compare the dimensions by calculating the volume. However, since this is a retrospective study, it is no longer possible to accurately assess the irregular volume of a tumor. In addition, the US and pathology data for this large sample study were obtained by different radiologists and pathologists, although they were all senior doctors. In addition, pathological sampling methods still need to be improved. Finally, other factors may be related to the measurement of tumor size, such as whether a core biopsy was performed prior to surgery, and the stiffness of the tumor. Further prospective studies are necessary. 


\section{Conclusions}

The use of US inevitably leads to an underestimation of the extent of the disease. Underestimated size is affected by pathological type, molecular subtype and histological size. The pathological results of the core biopsy help predict tumor size before accurate breast cancer diagnosis.

\section{Acknowledgements}

This work was supported in part by the National Natural Science Foundation of China (81572607, 81572595, 81502299 , and 81502286); the Natural Science Foundation of Jiangsu Province (BK2011853, BK2011855 and BK20141023); the Program for Development of Innovative Research Team in the First Affiliated Hospital of NJMU (IRT-008) and a project Funded by the Priority Academic Program Development of Jiangsu higher Education Institutions (PAPD).

\section{Conflict of interest: none}

\section{References}

1. Zhou W, Zha X, Liu X, et al. US-guided Percutaneous Microwave Coagulation of Small Breast Cancers: A Clinical Study. Radiology 2012;263:364-373.

2. Gruber IV, Rueckert M, Kagan KO, et al. Measurement of tumour size with mammography, sonography and magnetic resonance imaging as compared to histological tumour size in primary breast cancer. BMC Cancer 2013; 13:328.

3. Doddi S, Singhal T, Maghsoudi S, Sinha P, Desai AJ. Preoperative ultrasound for breast tumour measurements: is there potential for mismanagement? G Chir 2013;34:610.

4. Shoma A, Moutamed A, Ameen M, Abdelwahab A. Ultrasound for accurate measurement of invasive breast cancer tumor size. Breast J 2006;12:252-256.

5. Snelling JD, Abdullah N, Brown G, King DM, Moskovic E, Gui GP. Measurement of tumour size in case selection for breast cancer therapy by clinical assessment and ultrasound. Eur J Surg Oncol 2004;30:5-9.

6. Madjar H, Ladner HA, Sauerbrei W, Oberstein A, Prompeler H, Pfleiderer A. Preoperative staging of breast cancer by palpation, mammography and high-resolution ultrasound. Ultrasound Obstet Gynecol 1993;3:185-190.

7. Berg WA, Gutierrez L, NessAiver MS, et al. Diagnostic accuracy of mammography, clinical examination, US, and MR imaging in preoperative assessment of breast cancer. Radiology 2004;233:830-849.

8. Lee JW, Han W, Ko E, et al. Sonographic lesion size of ductal carcinoma in situ as a preoperative predictor for the presence of an invasive focus. J Surg Oncol 2008;98:1520.
9. Zhou W, Wang R, Liu X, et al. Ultrasound-guided microwave ablation: a promising tool in management of benign breast tumours. Int J Hyperthermia 2017;33:263-270.

10. Hashimoto H, Suzuki M, Oshida M, et al. Quantitative ultrasound as a predictor of node metastases and prognosis in patients with breast cancer. Breast Cancer 2000;7:241-246.

11. Chang RF, Hou YL, Huang CS, Chen JH, Chang JM, Moon WK. Automatic detection of microcalcifications in breast ultrasound. Med Phys 2013;40:102901.

12. Le Doussal V, Tubiana-Hulin M, Friedman S, Hacene K, Spyratos F, Brunet M. Prognostic value of histologic grade nuclear components of Scarff-Bloom-Richardson (SBR). An improved score modification based on a multivariate analysis of 1262 invasive ductal breast carcinomas. Cancer 1989;64:1914-1921.

13. Elston CW, Ellis IO. Pathological prognostic factors in breast cancer. I. The value of histological grade in breast cancer: experience from a large study with long-term follow-up. Histopathology 1991;19:403-410.

14. Yang WT, Tse GM. Sonographic, mammographic, and histopathologic correlation of symptomatic ductal carcinoma in situ. AJR Am J Roentgenol 2004;182:101-110.

15. Pritt B, Ashikaga T, Oppenheimer RG, Weaver DL. Influence of breast cancer histology on the relationship between ultrasound and pathology tumor size measurements. Mod Pathol 2004;17:905-910.

16. Satake H, Shimamoto K, Sawaki A, et al. Role of ultrasonography in the detection of intraductal spread of breast cancer: correlation with pathologic findings, mammography and MR imaging. Eur Radiol 2000;10:1726-1732.

17. Perou CM, Sorlie T, Eisen MB, et al. Molecular portraits of human breast tumours. Nature 2000;406:747-752.

18. Wojcinski S, Stefanidou N, Hillemanns P, Degenhardt F. The biology of malignant breast tumors has an impact on the presentation in ultrasound: an analysis of 315 cases. BMC Womens Health 2013;13:47.

19. Chen ST, Kuo SJ, Wu HK, Chen LS, Chen DR. Power Doppler breast ultrasound: association of vascularization and $\mathrm{ER} / \mathrm{c}$-erbB-2 co-expression in invasive breast carcinoma. Breast Cancer 2013; 20:152-158.

20. Dogan BE, Gonzalez-Angulo AM, Gilcrease M, Dryden MJ, Yang WT. Multimodality imaging of triple receptornegative tumors with mammography, ultrasound, and MRI. AJR Am J Roentgenol 2010;194:1160-1166.

21. Ko ES, Lee BH, Kim HA, Noh WC, Kim MS, Lee SA. Triple-negative breast cancer: correlation between imaging and pathological findings. Eur Radiol 2010;20:1111-1117.

22. Foulkes WD, Smith IE, Reis-Filho JS. Triple-negative breast cancer. N Engl J Med 2010;363:1938-1948.

23. Senkus E, Kyriakides S, Ohno S, et al. Primary breast cancer: ESMO Clinical Practice Guidelines for diagnosis, treatment and follow-up. Ann Oncol 2015;26 Suppl 5:v8v30.

24. Jackman RJ, Burbank F, Parker SH, et al. Atypical ductal hyperplasia diagnosed at stereotactic breast biopsy: improved reliability with 14-gauge, directional, vacuum-assisted biopsy. Radiology 1997;204:485-488. 